\title{
Suizidprävention: Wir brauchen bessere Netzwerke!
}

Martin Eichhorn, Sekretär SGKS

Information und Anmeldung: Barbara Weil IPSILON

Geschäftsleitung

Elfenstrasse 18

CH-3000 Bern 16

Tel. 0313591108

Internet: www.ipsilon.ch
Suizid und suizidales Verhalten ist häufig. Jede 100. Frau und jeder 30. Mann stirbt in der Schweiz durch Suizid. Jeder 10. Einwohner der Schweiz ist einmal wegen eines Suizidversuches in medizinischer Behandlung. Die Dunkelziffer der Suizidversuche ist gerade bei Jugendlichen hoch und Suizidgedanken müssen aufgrund der Häufigkeit schon als «normal» angesehen werden.

Suizid und suizidales Verhalten sind komplex. Oder als Frage formuliert: Wie entsteht aus persönlicher Disposition unter Einfluss von sozialen Faktoren suizidales Handeln?

Am 30. September 2004 führt die Schweizerische Gesellschaft für Krisenintervention und Suizidprophylaxe (SGKS) in Biel eine Fortbildungsveranstaltung durch, die sich zum Ziel setzt, der Komplexität der Suizidthematik eine differenzierte Sichtweise der (vernetzten) Interventionsmöglichkeiten entgegenzusetzen.

Krisenintervention wird nur zu einem Bruchteil durch das medizinische Versorgungssystem geleistet. In verschiedenen Vorträgen werden Hilfestellungen durch Internet- und Telefonberatung, in der Schule, durch die Polizei und andere vorgestellt. Diese Referate werden durch eine Posterausstellung ergänzt, in der laufende Initiativen und Projekte zur Suizidprävention vorgestellt werden.

Wie können wir voneinander lernen? Gerade in einem Gebiet, in dem so viele Mythen vorhanden sind, müssen wir uns mit dem Wissen aus allen Richtungen auseinandersetzen. Mit dem Erkennen einer Depression allein werden wir der Komplexität sicher nicht gerecht. Wir müssen uns vertieftes Wissen für die einzelnen Gruppen von suizidalen Menschen aneignen. So haben suizidales Verhalten von Männern und Frauen, Jungen und Alten, Migranten und Alteingesessenen andere Geschichten und andere Hintergründe. Es stellt sich nicht nur die Frage, wie wir die Krisenintervention gestalten, sondern auch die Frage, wie wir diese Menschen in Not erreichen können.
Wie können wir uns besser oder überhaupt erst vernetzen? Der erste Schritt zum Vernetzen ist das Kennenlernen. Das Akzeptieren, dass der andere Zugang zwar anderes, aber nicht falsch, sondern ergänzend ist.

Prof. Jean-Louis Terra wird aus seiner Erfahrung mit Netzwerkbildung in der Suizidprävention in Frankreich berichten, um damit eine Diskussion der Teilnehmer anzuregen. Wie können wir den Netzwerkgedanken in der Schweiz in die Praxis umsetzen? Wir erhoffen uns wertvolle Impulse in den nachmittäglichen Gruppenarbeiten. Sicher werden durch informelle Kontakte erste Netzwerkknoten geknüpft werden können. Das längerfristige Ziel muss in einer institutionalisierten Zusammenarbeit liegen. Eine wichtige und durchführbare Idee auf diesem Weg wäre zum Beispiel der kontinuierliche Einbezug von Fachleuten ausserhalb des eigenen Gartens bei der Fort- und Weiterbildung. Dass Netzwerke in der Suizidprävention erfolgreich sein können, zeigt sich im aktuellen Bündnis gegen Depression in Nürnberg. Wir sind überzeugt, dass die Vernetzung auch in der Schweiz eine zentrale Rolle bei allen Bemühungen zur Reduktion von suizidalem Verhalten spielen muss.

Aus diesem Grund freut sich die SGKS, die Tagung mit IPSILON - Initiative zur Prävention von Suizid in der Schweiz - zusammen durchführen zu können. IPSILON nimmt auf Ebene von Vereinen und Organisationen die Idee der Vernetzung auf, die auf Ebene von Einzelmitgliedern vor Jahren zur Gründung der SGKS geführt hat.

In ihrem Alltag sind Sie als Ärztin oder Arzt, aber auch privat, immer wieder mit suizidalem Verhalten konfrontiert. Sie leisten mit Ihrem Wissen einen wichtigen Beitrag zur Suizidprävention. Trotzdem stossen wir dauernd an unsere Grenzen. Wir möchten Sie einladen, an der Tagung teilzunehmen, um die Grenzen ein klein wenig auszuweiten. 